\title{
Pediatric head injury — an opportunity to make a difference in the global burden of disease
}

\author{
Anthony A. Figaji ${ }^{1}$
}

(C) Springer-Verlag GmbH Germany 2017

Dear Colleagues,

This special annual issue of Child's Nervous System covers some of the most important current topics in pediatric neurotrauma, extending from preclinical to clinical science, mild to severe traumatic brain injury (TBI), diagnosis to treatment, and principles to practice.

These are important topics and I would encourage you to read all the articles critically. We hope that these papers will stimulate your thinking, change your practice, and inspire new research ideas. This is much needed in our community. It is a sad reality that not all neurosurgeons have remained actively engaged with neurotrauma, a great pity for many reasons. First, the management of neurotrauma is the origin of our specialty, as evidenced from skulls of more than 4000 years ago. Second, it remains at the heart of our specialty based on the shear burden of disease worldwide. Even though most TBI occurs in low- and middle-income countries, still in highincome countries it remains the leading cause of death and disability in children and young adults. As such, it remains the most common condition in which we can make a meaningful difference to patient outcomes all over the world. Third, neurotrauma used to be at the forefront of neuroscience. Today, we neglect great opportunities on our doorstep to advance our understanding of the brain. In my opinion, neurotrauma is the most common, naturally occurring experimental condition of the brain, and we have yet much to learn from it.

Anthony A. Figaji

anthony.figaji@uct.ac.za

1 Paediatric Neurosurgery and Clinical Neurosciences Institute, University of Cape Town and Red Cross Children's Hospital, Cape Town, South Africa
Neurosurgeons must continue to be at the forefront of this great endeavor - to study the brain with the mind of a scientist, to adapt our treatments with the hands of a surgeon, and ultimately to make a difference in the lives of our patients with the heart of a humanitarian. To stimulate this enthusiasm, we have collected observations and insights from a diverse group of researchers and clinicians-basic scientists, surgeons, pediatricians, neurologists, and intensivists - all interested in advancing the art and science of head injury management.

Starting with preclinical insights, Patrick Kochanek and his team lead one of the most advanced and prestigious centers for understanding basic mechanisms of brain injury. Their overview is a window into the state of preclinical science of TBI and how it may inform our thinking in the clinic. Michael Bell and colleagues highlight the inadequacies of current guidelines for pediatric TBI care and summarize their very impressive work in developing a network of centers to work together and successfully complete the ADAPT Trial - a comparative effectiveness trial which is the first international multicenter trial on pediatric head injury management, funded by the National Institutes for Health. Theirs will be a remarkable contribution not only to the current practice of pediatric head injury management but also the foundation of future trials in this field.

Concussion has rapidly become a hot topic in neurosurgery, medicine, and society at large, filled with anxieties, uncertainties, and controversies. Chris Giza is one of the leading researchers in the field of concussion and a frequent contributor to international guidelines. He and Kerrigan update us on current knowledge of concussion and its implications for care. Continuing on the theme of mild TBI, there is always much discussion about when to perform head CT scanningSinghal and Singh summarize current thinking and what this means for your practice. 
Dennis et al. are the multidisciplinary neuroimaging team from UCLA and USC whose article summarizes progress and potential for advanced MRI techniques to reveal more about the injured brain, as a tool to understand, guide therapies, and prognosticate.

Jose Pineda and his team give us an overview of brain metabolism in pediatric TBI, a critically important subject very different from adult TBI, and discuss methods of monitoring and imaging to assist our understanding of this. Mayumi Prins addresses the complexities of glucose as a substrate for metabolism in the brain and its implications for glycemic control in pediatric TBI.

Donelly, Young, and Brady summarize the difficult issue of autoregulation in pediatric TBI, an important physiological function that has critical implications for bedside decisionmaking with respect to the management of blood pressure, intracranial pressure, and cerebral perfusion.

Naomi Ketharanathan and her colleagues from Rotterdam discuss analgesia and sedation in pediatric TBI, an essential component of our intensive care management for which we have surprisingly little data and widely divergent practices.

Matthieu Vinchon tackles the thorny issue of nonaccidental injury in children, a pediatric condition that perhaps more than any other reflects our troubled society, and for which there are many dangerous pitfalls for the social worker, clinician, pathologist, and lawyer.

Our team in Cape Town proposes the philosophy and practice of an individualized, targeted approach to pediatric TBI care. These are principles being applied in most fields of Medicine, recognizing the substantial differences between patients and their responses to treatment.

Another hot topic, and controversial one, is decompressive craniectomy in TBI. There is emerging evidence - and conflicted opinion - in adult TBI (without much resolution as yet), but still much less evidence to guide standardized care in children. This has stimulated a trio of related articles for this Issue. The team from Cambridge, hot on the heels of completing the recent RESCUE ICP trial in adults, has summarized the subject with reference to children. Our French colleagues examine the procedure with reference to the commonly encountered problems of cerebrospinal fluid disturbances. And our colleagues from Rome address the tricky challenges of reconstructive surgery after craniectomy in children. It is easy to take the bone off; putting it back may need more skill.

Takashi Araki and colleagues explore the issues of determining brain death in children from a Japanese perspective. It is important to remember that societal and physician views are as important as the precise issues of brain death criteria and its implications for transplant medicine; there are important geographical and cultural issues that we must consider.

Finally, what happens after we have done all we can to save a life and minimize neurological injury? Schrieff-Elson and Rohlwink lead a discussion on post-TBI rehabilitation in children, a subject often neglected by surgeons, but which has massive potential to improve long-term functional outcomes, ultimately what we all want to achieve.

So, we hope that you will be as excited about this collection of articles as I and all the authors involved have been about preparing and presenting them to you. We hope these contributions will stimulate thinking, debate, and communication between like minds. Knowing all of these authors, I am certain that they would welcome comment and questions about their work, and will be open to ideas for future collaborations. We need this open exchange of views to advance the field of pediatric neurotrauma research and practice. It is one of our best opportunities to make a meaningful difference to the global burden of disease, and to our very next patient.

\section{Compliance with ethical standards}

Conflict of interests I have no conflicts of interest. 